\title{
Differential effectiveness of berry polyphenols as anti-giardial agents
}

\author{
J.-P. ANTHONY ${ }^{1,2} \dagger$, L. FYFE ${ }^{1}$, D. STEWART ${ }^{3}$ and G. J. MCDOUGALL ${ }^{3} *$ \\ ${ }^{1}$ Dietetics, Nutrition and Biological Sciences, Queen Margaret University, Clerwood Terrace, Edinburgh EH12 8TS, UK \\ ${ }^{2}$ Scottish Parasite Diagnostic Laboratory, House on the Hill Reference Laboratories, Stobhill Hospital, Balornock Road, \\ Glasgow G21 3UW, UK \\ ${ }^{3}$ Plant Products and Food Quality Programme, The Fames Hutton Institute, Invergowrie, Dundee DD2 5DA, UK
}

(Received 29 March 2011; revised 26 April 2011; accepted 29 April 2011)

SUMMARY

Following previous work on the anti-giardial effect of blueberry polyphenols, a range of polyphenol-rich extracts from berries and other fruits was screened for their ability to kill Giardia duodenalis, an intestinal parasite of humans. Polyphenolrich extracts were prepared from berries using solid-phase extraction and applied to trophozoites of Giardia duodenalis grown in vitro. All berry extracts caused inhibition at $166 \mu \mathrm{g}$ gallic acid equivalents (GAE)/ml phenol content but extracts from strawberry, arctic bramble, blackberry and cloudberry were as effective as the currently used drug, metronidazole, causing complete trophozoite mortality in vitro. Cloudberry extracts were found to be the most effective causing effectively complete trophozoite mortality at $66 \mu \mathrm{g} \mathrm{GAE} / \mathrm{ml}$. The polyphenol composition of the more effective berry extracts suggested that the presence of ellagitannins could be an important factor. However, the potency of cloudberry could be related to high ellagitannin content but also to the presence of substantial amounts of unconjugated $p$-coumaric acid and benzoic acid. These in vitro effects occur at concentrations easily achievable in the gut after berry ingestion and we discuss the likelihood that berry extracts could be effective anti-giardial agents in vivo.

Key words: berry, polyphenol, Giardia duodenalis, ellagitannin, trophozoite, mortality.

\section{INTRODUCTION}

Giardia duodenalis is a flagellated protozoan parasite that colonizes and reproduces in the human intestine, causing giardiasis, of which the most common symptom is serious diarrhoea. Effective drug treatment for $G$. duodenalis is available, using orally administered metronidazole, but there is evidence of resistance (e.g. Harris et al. 2001; Upcroft et al. 2006). In addition, side effects such as nausea and headaches combined with an unpalatable metallic taste reduce patient compliance. In poorer countries where giardiasis is endemic, this condition affects both child development and mortality and chemotherapy may be minimally effective due to continual re-infection from the environment and therefore novel drugs and treatments are sought (Rossignol, 2010).

Plants and plant products have been used as traditional remedies for various ailments in numerous countries, with little or no side effects (Jones,

* Corresponding author: Plant Products and Food Quality Programme, The James Hutton Institute, Invergowrie, Dundee DD2 5DA, UK. Fax: + 441382 562426. Tel: +441382 562731. E-mail: Gordon.mcdougall@hutton. ac.uk

† Current address: Solus Scientific Solutions, Royal College Building, R4.19, University of Strathclyde, 204 George Street, Glasgow G1 1XW, UK.
1996). Wild blueberries (Vaccinium myrtillus) and wild strawberries (Fragaria vesca) have been historically used to treat 'fluxes' (or florid diarrhoea) in the UK (see Anthony et al. 2007). The true causes of such diarrhoea/fluxes cannot be confirmed but certainly could be caused by enteropathogens (Cox, 2002) such as Giardia and Cryptosporidium.

The anti-microbial properties of plant products have been recognized for numerous years, and recently there has been renewed interest in berries and their phenolic constituents such as anthocyanins and ellagitannins (e.g. Nohynek et al. 2006). Polyphenol-rich berry extracts inhibited the growth of enteropathogenic bacteria such as Salmonella, Escherichia, Staphylococcus, Helicobacter, Bacillus, Clostridium and Campylobacter species, and were bacteriostatic for 3 Staphylococcus aureus strains (Puupponen-Pimia et al. 2005).

Trophozoites from infective cysts infect the duodenum, attaching onto enterocytes via a combination of hydrodynamic forces and their ventral 'sucking' disc and through lectins specific for D-glucosyl and D-mannose residues (Inge et al. 1988). Berry extracts may inhibit the adhesion and colonization of Giardia to human enterocytes. Proanthocyanidins found in cranberry juice (Vaccinium macrocarpon, and in other Vaccinium species) have potent bacterial anti-adhesion activity (Howell et al. 2005) and the proanthocyanidin,

Parasitology (2011), 138, 1110-1116. C Cambridge University Press 2011. The online version of this article is published within an Open Access environment subject to the conditions of the Creative Commons Attribution-NonCommercial-ShareAlike licence $<$ http://creative commons.org/licenses/by-nc-sa/2.5/>. The written permission of Cambridge University Press must be obtained for commercial re-use. 
Geranin D, from Geranium niveum has potent antigiardial activity (Calzada et al. 2001) Plant products have been demonstrated to possess anti-protozoan effects, but relatively little is known. Garlic extract inhibits $G$. duodenalis trophozoite growth in vitro (Lun et al. 1994) and treatment with garlic extract can reduce clinical signs and symptoms more rapidly than metronidazole, the current drug of choice (Soffar and Mokhtar, 1991). Whole garlic extract co-cultured with trophozoites causes internalization of flagella and ventral disc fragmentation (Harris et al. 2000; Anthony et al. 2005). Isoflavones, especially formononetin from Dalbergia frutescens, have potent anti-giardial activity in vitro (Khan et al. 2000) with a possible mode of action being the detachment of Giardia trophozoites in vitro and ex vivo through the inhibition of flagellar motility (Lauwaet et al. 2010).

Previous work investigated a pressed extract of blueberries, a commercial drink made from this extract and a polyphenolic-rich extract for their ability to influence $G$. duodenalis trophozoite viability in vitro and confirmed the effectiveness of the polyphenolic components (Anthony et al. 2007). This study examines the effects of polyphenol-rich extracts from a range of berry species with disparate composition on Giardia viability in vitro.

\section{MATERIALS AND METHODS}

\section{Extraction of berries}

Blackcurrants (Ribes nigrum L. variety 8982-6) were obtained from Bradenham Hall, Norfolk, UK and blueberries (Vaccinium myrtillus L. variety Berkeley) were grown at SCRI. Cloudberries (Rubus chamaemorus L.), arctic bramble (Rubus stellatus $\times R$. arcticus), lingonberries (Vaccinium vitis-idaea), sea buckthorn (Hippophae rhamnoides) and rowan berries (Sorbus aucuparia L. c.v. Sahharnaja) were obtained from Dr Harri Kokko, University of Kuopio. Pomegranates (Punica granatum L.) were purchased from a local supermarket. Strawberries (Fragaria ananassa variety Elsanta) were obtained from local farmers.

The berries were extracted by the protocol outlined previously (McDougall et al. 2009) with minor changes. Briefly, the berries were homogenized in an equal volume to weight of $0.2 \%(\mathrm{v} / \mathrm{v})$ acetic acid in $50 \%$ acetonitrile/ultra-pure water (UPW) in a Waring blender ( 5 times for $15 \mathrm{sec}$ on full power). The extract was filtered through tripled muslin then centrifuged at $2800 \mathrm{~g}$ for $10 \mathrm{~min}$ at $4{ }^{\circ} \mathrm{C}$ to remove suspended polysaccharides.

The extracts were each applied to separate C18 solid-phase extraction (SPE) units (Strata C18-E, GIGA units, $10 \mathrm{~g}$ capacity Phenomenex Ltd, Macclesfield, UK) pre-washed in $0.2 \%(\mathrm{v} / \mathrm{v})$ formic acid in acetonitrile then pre-equilibrated in $0.2 \%$ (v/v) formic acid in UPW. The unbound material, which contained the free sugars, organic acids and vitamin C, was collected. The units were washed with 3 column volumes of UPW then the polyphenolenriched bound extracts eluted with $0.1 \%(\mathrm{v} / \mathrm{v})$ formic acid in acetonitrile.

Phenol content was measured using a modified Folin-Ciocalteu method (Deighton et al. 2000) and quantified as gallic acid equivalents (GAE). Anthocyanin content was measured using the 2 -wavelength method outlined previously (Deighton et al. 2000). The C18-bound extracts were evaporated to dryness in a Speed-Vac (Thermo-Finnegan Ltd) as required.

\section{Culture of G. duodenalis trophozoites}

Trophozoites of the BVM strain of $G$. duodenalis were maintained axenically in flat-sided $110 \mathrm{~mm} \times$ $16 \mathrm{~mm}$ culture tubes at $37^{\circ} \mathrm{C}$, in a modified TYIS-33 medium (Anthony et al. 2007) supplemented with $10 \%(\mathrm{v} / \mathrm{v})$ heat-inactivated foetal bovine serum. Subculturing was performed routinely at $72-96 \mathrm{~h}$ intervals. After $72 \mathrm{~h}$ of culture, trophozoites were harvested by chilling culture vessels in iced water for $20 \mathrm{~min}$. For addition to G. duodenalis cultures, aliquots of dried berry polyphenol extracts were reconstituted in TYIS-33 at a concentration of $2 \mathrm{mg} \mathrm{ml}^{-1}$ then passed through a sterile $0.22 \mu \mathrm{m}$ filter (Sartorius).

Experiments were performed in sterile, 96-well microtitre plates covered with plate-sealer film and lids. To $100 \mu \mathrm{l}$ of trophozoite culture $\left(\sim 2.7 \times 10^{4}\right.$ trophozoites) $200 \mu \mathrm{l}$ of berry extract diluted in TYI-S-33 was added to give suitable final concentrations. The microtitre plates were sealed and incubated for $24 \mathrm{~h}$ at $37^{\circ} \mathrm{C}$, after which the trophozoites were enumerated. The positive control consisted of $2.7 \times 10^{4}$ trophozoites in $300 \mu$ of TYI-S-33 containing $67 \mu \mathrm{g} \mathrm{ml}^{-1}$ metronidazole (found to be the minimal inhibitory concentration within this system that killed $100 \%$ of trophozoites) and the negative controls consisted of $2.7 \times 10^{4}$ trophozoites in $300 \mu \mathrm{l}$ of TYI-S-33 in the absence of berry extracts or metronidazole. All assays were carried out in triplicate.

Trophozoite enumeration was performed using an improved Neubauer haemocytometer and trophozoite viability determined by Trypan blue inclusion/ exclusion. Briefly, trophozoites were harvested from wells by chilling the plate on iced water for $20 \mathrm{~min}$ and the contents of triplicate wells were combined into $1.5 \mathrm{ml}$ microcentrifuge tubes, and then centrifuged (10 sec, bench top centrifuge 5410, Eppendorf $\mathrm{GmbH}$, Germany). The supernatant was aspirated to waste and the pellet resuspended in $100 \mu \mathrm{l}$ of TYI-S-33 and $100 \mu$ of $0.4 \%$ Trypan blue solution (Gibco, UK) and mixed thoroughly. After mixing, $10 \mu \mathrm{l}$ were dispensed into both chambers of an 


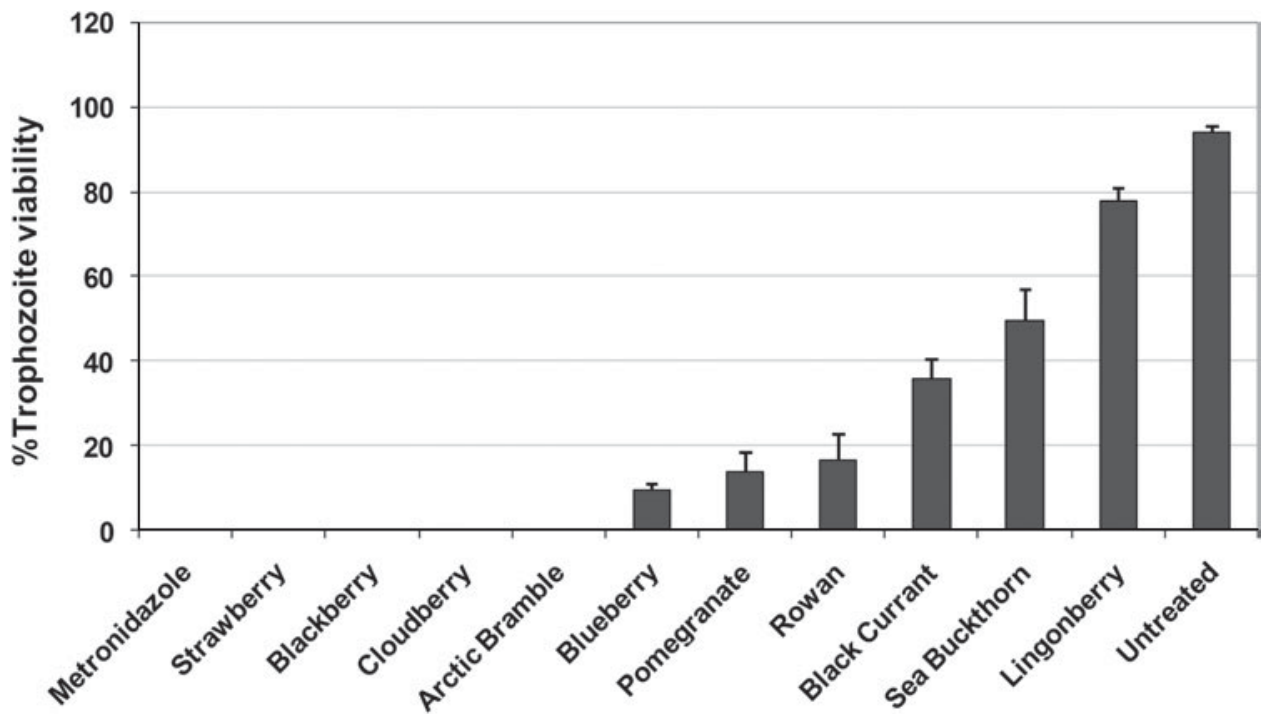

Fig. 1. Effect of berry extracts on Giardia viability. Trophozoites $\left(2.7 \times 10^{4}\right.$ trophozoites per well) were incubated for $24 \mathrm{~h}$ in the presence or absence of berry polyphenol extracts at $50 \mu \mathrm{g}$ GAE per well. Untreated trophozoites and metronidazole $\left(67 \mu \mathrm{g} \mathrm{ml}^{-1}\right)$ treated trophozoites were used as controls. All values are averages of triplicate experiments \pm standard error. All berry extracts significantly reduced the survival of $G$. duodenalis trophozoites when compared to untreated trophozoites $(P \leqslant 0.05)$.

improved Neubauer haemocytometer, allowed to settle $(30-60 \mathrm{sec})$ then assessed.

Trophozoite viability was determined under bright-field microscopy at a total magnification of $\times 400$ using the following formula: $(\mathrm{L} \div(\mathrm{L}+\mathrm{D})) \times 100$ where $L$ is the number of live trophozoites (unstained) and D the number of dead trophozoites (stained with Trypan blue) with 3 counts of 100-200 trophozoites being taken and repeated twice.

\section{Liquid Chromatography Mass Spectrometry (LC-MS) analysis}

Samples containing $20 \mu \mathrm{g}$ phenols (GAE) were analysed on an LCQ-Deca system, comprising Surveyor auto-sampler, pump and photo-diode array detector (PDAD) and a ThermoFinnigan mass spectrometer iontrap as described previously (McDougall et al. 2009). The PDAD scanned discrete channels at $280 \mathrm{~nm}, 365 \mathrm{~nm}$ and $520 \mathrm{~nm}$. The samples $(20 \mu \mathrm{l})$ were applied to a $\mathrm{C}-18$ column (Synergi Hydro C18 with polar-end capping, $4.6 \mathrm{~mm} \times 150 \mathrm{~mm}$, Phenomenex Ltd, UK) and eluted over a gradient of $5 \%$ acetonitrile $(0.5 \%$ formic acid $)$ to $30 \%$ acetonitrile $(0.5 \%$ formic acid) over $60 \mathrm{~min}$ at a rate of $400 \mu \mathrm{l} / \mathrm{min}$. The LCQ-Deca LC-MS was fitted with an ESI (electrospray ionization) interface and analysed the samples in positive and negative ion mode. There were 2 scan events; full scan analysis followed by data dependent MS/MS of most intense ions using collision energies (source voltage) of $45 \%$. The capillary temperature was set at $250^{\circ} \mathrm{C}$, with sheath gas at $60 \mathrm{psi}$ and auxiliary gas at $15 \mathrm{psi}$. The MS was tuned against solutions of ellagic acid (negative mode) and cyanidin-3-O-glucoside (positive mode). Only negative mode data are shown.
Putative identifications of polyphenols are based on previous work in cloudberry (McDougall et al. 2008, 2009) and previous literature (Mullen et al. 2003; Hager et al. 2008; Gasperotti et al. 2010). Benzoic acid, $p$-coumaric acid and ellagic acid were employed as standards to confirm the identity of peaks. Peaks identified as ellagitannins were quantified using the MS system (Xcalibur) software and their PDA peak areas summed. This figure was divided by the total peak area of all polyphenol peaks to give an estimate of relative ellagitannin content. This estimate was carried out on 3 replicate injections of the arctic bramble and the cloudberry samples. This comparison was facilitated as the same ellagitannin peaks were largely present in cloudberry and arctic bramble.

\section{RESULTS}

Strawberry, blackberry, cloudberry and arctic bramble were as effective in killing Giardia as the positive control, metronidazole, at $50 \mu \mathrm{g}$ GAE/well (Fig. 1). These effects were not due to changes in $\mathrm{pH}$ as the polyphenol-enriched extracts were effectively devoid of organic acids and did not alter the $\mathrm{pH}$ of the medium. There was no correlation between anthocyanin content and effectiveness as both cloudberry $(<0.1 \%)$ and blackberry $(58.0 \%)$ anthocyanin content; (see Supplementary data, Table S1, Online version only) caused complete trophozoite killing at $50 \mu \mathrm{g} \mathrm{GAE}_{\text {well }}{ }^{-1}$.

The most effective berry extracts were re-assayed at lower concentrations to assess dose-response effects (Fig. 2). This revealed an order of effectiveness of blueberry $<$ strawberry $<$ blackberry $=$ arctic bramble $<$ cloudberry. Cloudberry extracts were most 


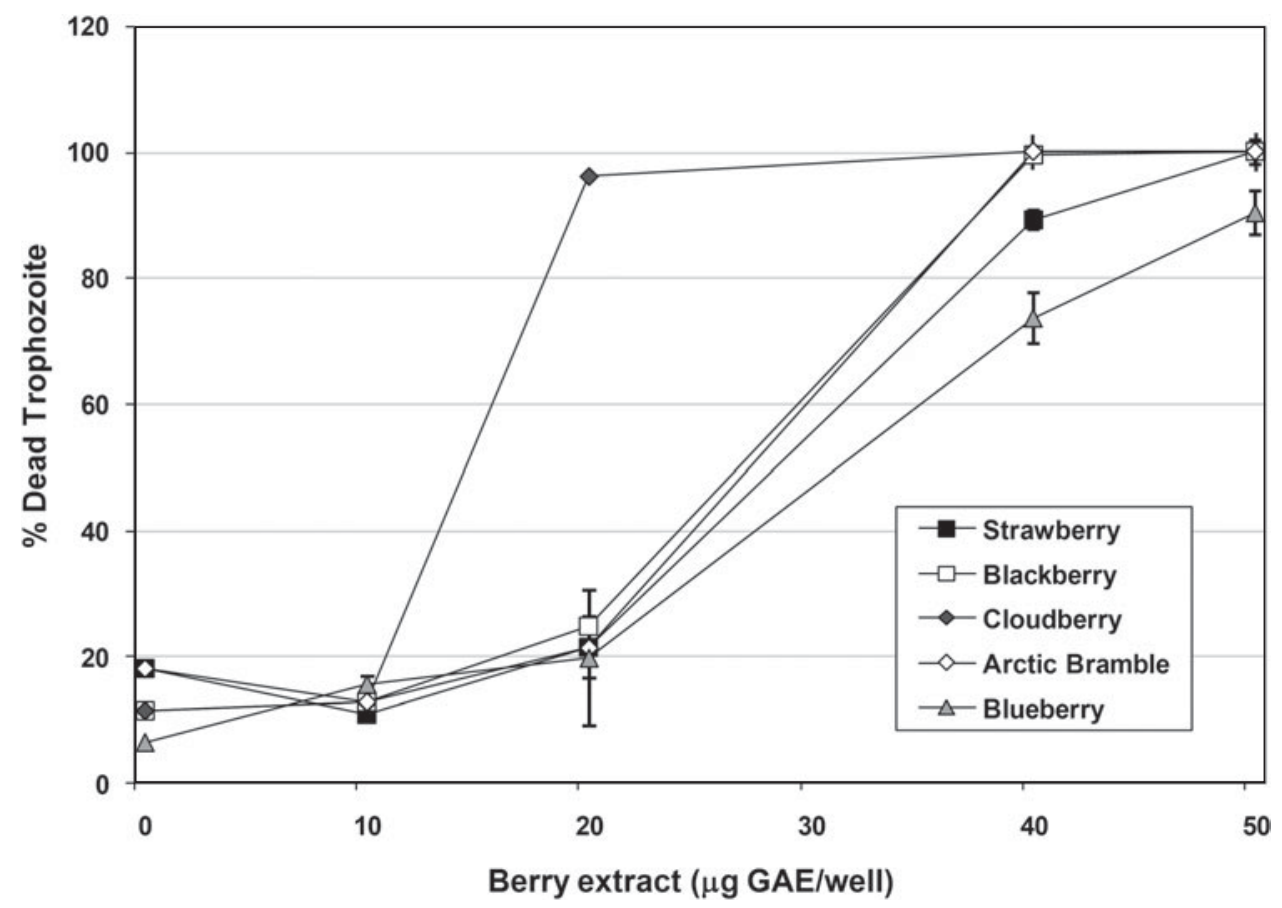

Fig. 2. Dose effects of selected berry extracts on Giardia viability. Trophozoites $\left(2.7 \times 10^{4}\right.$ trophozoites per well $)$ were incubated for $24 \mathrm{~h}$ in the presence or absence of berry polyphenol extracts at various concentrations. Untreated trophozoites and metronidazole $\left(67 \mu \mathrm{g} \mathrm{ml}^{-1}\right)$ treated trophozoites were used as controls. Control metronidazole treatments caused $100 \%$ trophozoite mortality with a replication error averaging at $2.6 \%$ and all berry polyphenol extracts caused a dose-dependent reduction in trophozoite viability.

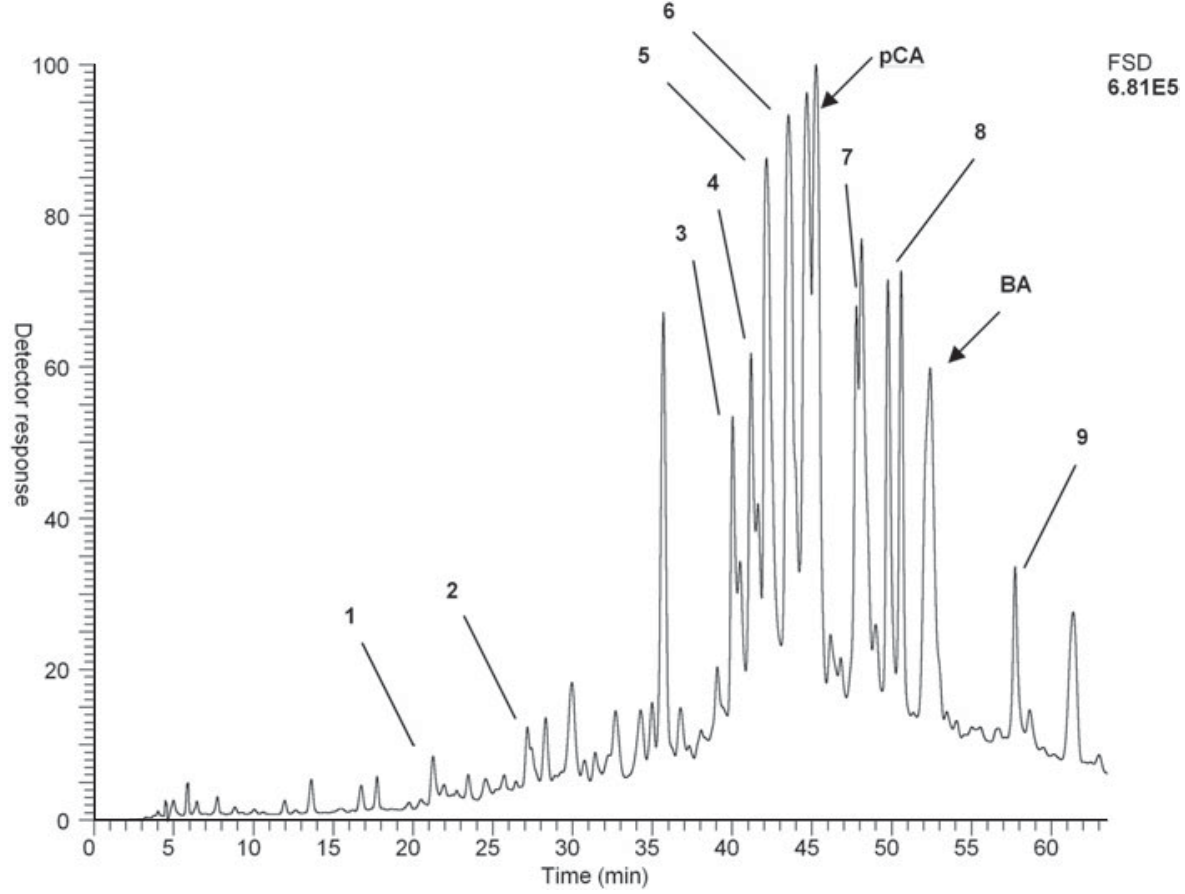

Fig. 3. LC-MS trace of cloudberry extract. All peak assignments relate to Table 1.pCA, p-coumaric acid and BA, benzoic acid. The figure in the top right corner is the full-scale deflection value for the PDA.

effective and caused high levels of killing at $20 \mu \mathrm{g}$ $\mathrm{GAE} \mathrm{well}^{-1}$ when the other extracts had lost activity.

LC-MS analysis (Fig. 3) confirmed that ellagitannins were the predominant polyphenolic components in the cloudberry extracts (Table 1) but also identified significant amounts of benzoic acid and $p$-coumaric acid. As an example, the mass spectral properties leading to the identification of peak 6 as Sanguiin H6 are discussed in more detail in supplementary data (Supplementary Fig. S1, Online version only). Smaller amounts of flavonol derivatives (e.g. quercetin-3-O-glucuronide) could also be 
Table 1. Putative identification of ellagitannin peaks in cloudberry extract

(All MS data are from negative mode ionization. The major signal is given in bold. Putative identifications are based on previous work on cloudberry (McDougall et al. 2008, 2009) and previous literature (Mullen et al. 2003; Gasperotti et al. 2010; Hager et al. 2008). More detailed information about the identification of peak 6 as sanguiin H6 is shown in the Supplementary data (Fig. S1), Online version only.)

\begin{tabular}{|c|c|c|c|c|c|}
\hline Peak no. & $\begin{array}{l}\text { Retention } \\
\text { time }\end{array}$ & $\begin{array}{l}\text { PDA } \\
\max .\end{array}$ & $m / z[\mathrm{M}-\mathrm{H}]$ & $\mathrm{MS}^{2}$ & Putative identity \\
\hline 1 & 21.25 & 275 & $\mathbf{7 8 3}, 481,301$ & $481,301,275$ & Pendunculagin isomer \\
\hline 2 & 28.31 & 275 & $\mathbf{6 3 3}, 301,275$ & 301 & Galloyl-HHDP-glucose \\
\hline 3 Front & 40.03 & 275 & 1869 & $\mathbf{1 5 6 7}, 1265,1235,1103,933$ & Sanguiin H6 isomer \\
\hline Rear & & & 1717,933 & $\mathbf{1 4 1 5}, 1113,933,783$ & $\begin{array}{l}\text { Sanguiin H-6 } \\
\text { without gallic moiety }\end{array}$ \\
\hline 4 Front & 41.17 & 275 & 1567 & $\mathbf{1 2 6 5}, 1103,935,801,631$ & Sanguiin H10 \\
\hline Rear & & & 933 & $897,631, \mathbf{4 5 1}, 315$ & Unknown ellagitannin \\
\hline 5 & 42.15 & 275 & 1401 & Multiple & Lambertinian $\mathrm{C}$ \\
\hline 6 & 43.52 & 275 & 1869 & $1567,1265,1235,1103,933,631$ & Sanguiin H6 \\
\hline 7 & 47.88 & 275 & 935 & 898,633 & Galloyl-bis-HHDP glucose \\
\hline 8 & 49.85 & 370 & 301 & 301 & Ellagic acid \\
\hline 9 & 57.73 & 275 & $1085,783,301$ & $915, \mathbf{8 9 7}, 783,633,451$ & Unknown ellagitannin \\
\hline
\end{tabular}

identified. The ellagitannin content of the cloudberry extract was estimated at approximately $72.0 \pm 1.4 \%$ compared to $44.1 \pm 0.8 \%$ for the less effective extract from the related arctic bramble. Although this value is only an estimate (calculated from a ratio of the peak area due to ellagitannins and ellagic acid divided by the total peak area of all identified phenolic components), it certainly reflects the higher content of non-ellagitannin contents such as anthocyanins in arctic bramble (see Supplementary data, Table S1).

Indeed, the high ellagitannin content of cloudberry has been noted previously in this laboratory (McDougall et al. 2008) and in previous work (Kähkönen et al. 2001; Kopenen et al. 2007). For example, Kähkönen et al. (2001) estimated the ET content in different cloudberry samples that ranged from 65 to $88 \%$ of total phenol content. However, the ellagitannin content of arctic bramble has not been published previously.

\section{DISCUSSION}

Berry extracts have long been known to interfere with human microbial pathogens. One of the most widely known is the inhibitory effect of proanthocyanidins from cranberry (Howell et al. 2005), and lingonberry (Kontiokari et al. 2001), on bacterial urinary tract infections. Berry extracts rich in ellagitannins have also been noted as being particularly effective against human pathogenic micro-organisms (Heinonen et al. 2007) with cloudberry being the most effective against the widest range of bacteria and yeasts (Nohynek et al. 2006). This builds on work that outlined anti-microbial activity of ellagitannins from other plant sources (Latte and Kolodziej, 2000; Asres et al. 2001; Machado et al. 2002; Parashar et al. 2009, and reviewed by Yoshida et al. 2009).
Indeed, ellagitannin-rich extracts and purified ellagitannins have been reported to have effects on human protozoan parasites such as Trypanosoma (Shuaibu et al. 2008), Plasmodium species (Dell'Agli et al. 2009) and Leishmania species (Kolodziej and Kiderlen, 2005). Ellagic acid, which can be formed by ellagitannin degradation, has also been shown to be effective against Plasmodium species in vivo and in vitro (Soh et al. 2009). Ellagic acid was also identified as an active anti-giardial agent in Rubus coriifolius (Alanis et al. 2003). In addition, other studies have shown toxicity of ellagitannins against the nematode, C. elegans (Yamasaki et al. 2002).

Here we have shown that the polyphenol-rich extracts of strawberry, blackberry, cloudberry and arctic bramble were as effective as metronidazole in the in vitro killing of $G$. duodenalis trophozoites and that cloudberry proved to be the most effective. The minimum concentration of cloudberry phenolics found to be effective against Giardia trophozoites in this study was between 10 and $20 \mu \mathrm{g}$ GAE per well (which equates to $33-66 \mu \mathrm{g} \mathrm{ml}^{-1}$ ). Considering that cloudberries can provide around $150 \mathrm{mg} \mathrm{GAE} / 100 \mathrm{~g}$ fruit (approx. $1500 \mu \mathrm{g} \mathrm{ml}^{-1}$ : Kähkönen et al. 2001), the amount of phenolic material entering the small intestine could easily reach levels shown to be effective in vitro, even allowing for partial degradation in the stomach (Clifford and Scalbert, 2000). In studies with ileostomy volunteers, GonzalezBarrio et al. (2010) have shown that a large proportion of ingested raspberry polyphenols were recovered in ileal fluid and, moreover, about a quarter of the main ellagitannin component, Sanguiin H6, survived digestion in the upper gastrointestinal tract.

As well as having a high percentage of ellagitannins, cloudberries are notable for having substantial levels of unconjugated $p$-coumaric acid and benzoic 
acid (Maatta-Riihinen et al. 2004). p-Coumaric acid has been shown to have a weak effect on Giardia trophozoites (Calzada and Alanis, 2007) and the coumaric acid derivative (melilotoside) was identified as an active anti-giardial agent from Teloxys graveolens (Calzada et al. 2003). The presence of $p$-coumaric and benzoic acid may potentiate the effects on Giardia caused by the ellagitannins but perhaps by a different and synergistic mechanism (Fyfe et al. 1998; Puupponen-Pimiä et al. 2001). In addition, quercetin derivatives, also identified in cloudberry, may also contribute to anti-giardial activity (Amaral et al. 2006; Calzada and Alanis 2007).

In conclusion, this study has confirmed that berry polyphenols can influence Giardia survival in vitro and suggests that ellagitannins are most effective. Tannin-rich preparations may also have efficacious effects on diarrhoeal symptoms (Palombo, 2006) often associated with Giardia infection. Unlike many plant sources of anti-giardial agents (e.g. Amaral et al. 2006), berries are a natural and palatable foodstuff and therefore have few issues with toxicity, side effects or acceptance. However, further work is required to uncover whether these in vitro effects described here can be transferred to the in vivo situation and contribute to giardiasis treatment.

\section{ACKNOWLEDGEMENTS}

SCRI receives grant-in-aid from the Scottish Government Rural and Environment Research and Analysis Directorate (RERAD). In memoriam of Huw Vaughn Smith who sadly passed away in October 2010 and contributed greatly to parasitology and to the analysis presented in this manuscript.

\section{REFERENCES}

Alanis, A. D., Calzada, F., Cedillo-Rivera, R. and Meckes, M. (2003). Anti-protozoal activity of the constituents of Rubus coriffolius. Phytotherapy Research 17, 681-682

Amaral, F. M. M., Ribeiro, M. N. S., Barbosa-Filho, J.-M., Reis, A. S., Nascimento, F. R. F. and Macedo, R. O. (2006). Plants and chemical constituents with giardicidal activity. Brazilian Yournal of Pharmacognosy 16, 696-720.

Anthony, J.-P., Fyfe, L. and Smith, H. V. (2005). Plant essential oils - a resource for antiparasitic agents? Trends in Parasitology 21, 462-468.

Anthony, J.-P., Fyfe, L., Stewart, D., and McDougall, G. J. and Smith, H. V. (2007). The effect of blueberry extracts on Giardia duodenalis viability and spontaneous excystation of Cryptosporidium parvum oocysts, in vitro. Methods 42, 339-348.

Asres, K., Bucar, F., Edelsbrunner, S., Kartnig, T. and Hoger, G. (2001). Investigations on anti-mycobacterial activity of some Ethiopian medicinal plants. Phytotherapy Research 15, 323-326.

Calzada, F. and Alanís, A. D. (2007). Additional antiprotozoal flavonol glycosides of the aerial parts of Helianthemum glomeratum. Phytotherapy Research 21, 78-80.

Calzada, F., Cedillo-Rivera, R., Bye, R. and Mata, R. (2001). Geranins $\mathrm{C}$ and $\mathrm{D}$, additional new anti-protozoal A-type proanthocyanidins from Geranium niveum. Planta Medica 67, 677-680.

Calzada, F. C., Velazquez, R., Cedillo-Rivera, R. and Esquivel, B. (2003). Antiprotozoal activity of the constituents of Teloxys graveolens. Phytotherapy Research 17, 731-732.

Clifford, M. N. and Scalbert, A. (2000). Ellagitannins-nature, occurrence and dietary burden. Fournal of the Science of Food and Agriculture 80, $1118-1125$.
Cox, F. E. G. (2002). History of human parasitology. Clinical Microbiology Reviews 15, 595-612.

Deighton, N., Brennan, R., Finn, C. and Davies, H. V. (2000) Antioxidant properties of domesticated and wild Rubus species. Fournal of the Science of Food and Agriculture 80, 1307-1313.

Dell'Agli, M., Galli, G. V., Corbett, Y., Taramelli, D., Lucantoni, L., Habluetzel, A., Maschi, O., Caruso, D., Giavarini, F., Romeo, S., Bhattacharya, D. and Bosisio, E. (2009). Anti-plasmodial activity of Punica granatum L. fruit rind. Fournal of Ethnopharmacology 125, 279-285. Fyfe, L., Armstrong, F. and Stewart, J. (1998). Inhibition of Listeria monocytogenes and Salmonella enteriditis by combinations of plant oils and derivatives of benzoic acid: the development of synergistic antimicrobial combinations. International Yournal of Antimicrobial Agents 9, 195-199.

Gasperotti, M., Masuero, D., Vrhovsek, U., Guella, G. and Mattivi, F. (2010). Profiling and accurate quantification of Rubus ellagitannins and ellagic acid conjugates using direct UPLC-Q-TOF HDMS and HPLCDAD analysis. Fournal of Agricultural and Food Chemistry 58, 4602-4616. Gonzalez-Barrio, R., Borges, G., Mullen, W. and Crozier, A. (2010). Bioavailability of anthocyanins and ellagitannins following consumption of raspberries by healthy humans and subjects with an ileostomy. Fournal of Agricultural and Food Chemistry 58, 3933-3939.

Hager, T. J., Howard, L. R., Liyanage, R., Lay, J. O. and Prior, R. L. (2008). Ellagitannin composition of blackberry as determined by HPLC-ESI-MS and MALDI-TOF-MS. Fournal of Agricultural and Food Chemistry 56, 661-669.

Harris, J. C., Plummer, S., Turner, M. P. and Lloyd, D. (2000). The microaerophilic flagellate Giardia intestinalis: Allium sativum (garlic) is an effective antigiardial. Microbiology 146, 3119-3127.

Harris, J. C., Plummer, S. and Lloyd, D. (2001). Antigiardial drugs. Applied Microbiology and Biotechnology 57, 614-619.

Heinonen, M. (2007). Antioxidant activity and antimicrobial effect of berry phenolics - a Finnish perspective. Molecular Nutrition $\&$ Food Research 51, 684-691.

Howell, A. B., Reed, J.D., Krueger, C.D., Winterbottom, R., Cunningham, D. G. and Leahy, M. (2005). A-type cranberry proanthocyanidins and uropathogenic bacterial anti-adhesion activity. Phytochemistry 66, 2281-2291.

Inge, P. M., Edson, C. M. and Farthing, M. J. M. (1988). Attachment of Giardia lamblia to rat intestinal epithelial cells. Gut 29, 795-801.

Jones, F. A. (1996). Herbs - useful plants. Their role in history and today. European Yournal of Gastroenterology \& Hepatology 8, 1227-1231.

Kähkönen, M.P., Hopia, A.I. and Heinonen, M. (2001). Berry phenolics and their antioxidant activity. Fournal of Agricultural and Food Chemistry 49, 4076-4082.

Khan, I. A., Avery, M. A., Burandt, C. L., Goins, D. K., Mikell, J. R., Nash, T.E., Azadegan, A. and Walker, L. A. (2000). Anti-giardial activity of isoflavones from Dalbergia frutescens bark. Fournal of Natural Products 63, 1414-1416.

Kolodziej, H. and Kiderlen, A. F. (2005). Anti-leishmanial activity and immune modulatory effects of tannins and related compounds on Leishmania parasitised RAW 264.7 cells. Phytochemistry 66, 2056-2071.

Kontiokari, T., Sundqvist, K., Nuutinen, M., Pokka, T., Koskela, M., and Uhari, M. (2001). Randomised trial of cranberry-lingonberry juice and Lactobacillus GG drink for the prevention of urinary tract infections in women. British Medical fournal 322, 1571-1573.

Koponen, J. M., Happonen, A. M., Mattila, P. H. and Törrönen, A. R. (2007). Contents of anthocyanins and ellagitannins in selected foods consumed in Finland. Fournal of Agricultural and Food Chemistry 55, 1612-1619.

Latte, K. P. and Kolodziej, H. (2000). Antifungal effects of hydrolysable tannins and related compounds on dermatophytes, mould fungi and yeasts. Zeitschrift für Naturforschung C-A Yournal of Biosciences 55, 467-472.

Lauwaet, T., Andersen, Y., Van de Ven, L., Eckmann, L. and Gillin, F. D. (2010). Rapid detachment of Giardia lamblia trophozoites as a mechanism of antimicrobial action of the isoflavone formononetin. Fournal of Antimicrobial Chemotherapy 65, 531-534.

Lun, Z. R., Burri, C., Menzinger, M. and Kaminsky, R. (1994). Antiparasitic activity of diallyl trisulfide (Dasuansu) on human and animal pathogenic protozoa (Trypanosoma sp., Entamoeba histolytica and Giardia lamblia) in vitro. Annales de la Societe Belge de Medecine Tropicale 74, 51-59. Maatta-Riihinen, K. R., Kamal-Eldin, A. and Torronen, R. (2004). Identification and quantification of phenolic compounds in berries of Fragaria and Rubus species (family Roseaceae). Fournal of Agricultural and Food Chemistry 52, 6178-6187.

Machado, T.D., Leal, I. C. R., Amaral, A.C.F., dos Santos, K. R. N., da Silva, M. G. and Kuster, R. M. (2002). Antimicrobial ellagitannin of Punica granatum fruits. Fournal of the Brazilian Chemical Society 13, 606-610. 
McDougall, G. J., Kulkarni, N. N. and Stewart, D. (2009). Berry polyphenols inhibit pancreatic lipase activity in vitro. Food Chemistry $\mathbf{1 1 5}$, 193-199.

McDougall, G., Martinussen, I. and Stewart, D. (2008). Towards fruitful metabolomics: high throughput analyses of polyphenol composition in berries using direct infusion mass spectrometry. Fournal of Chromatography B 871, 362-369.

Mullen, W., Yokota, T., Lean, M. E. J. and Crozier, A. (2003). Analysis of ellagitannins and conjugates of ellagic acid and quercetin in raspberry fruits by LC-MS ${ }^{\mathrm{n}}$. Phytochemistry 64, 617-624.

Nohynek, L. J., Alakomi, H. L., Kähkönen, M.P., Heinonen, M. Helander, K. M., Oksman-Caldentey, K. M. and PuupponenPimiä, R.H. (2006). Berry phenolics: Antimicrobial properties and mechanisms of action against severe human pathogens. Nutrition and Cancer-an International fournal 54, 18-32.

Palombo, E. A. (2006). Phytochemicals from traditional medicinal plants used in the treatment of diarrhoea: modes of action and effects on intestinal function. Phytotherapy Research 20, 717-724.

Parashar, A., Gupta, C., Gupta, S. K. and Kumar, A. (2009) Antimicrobial ellagitannin from pomegranate (Punica granatum) fruits. International Fournal of Fruit Science 9, 226-231.

Puupponen-Pimiä, R., Nohynek, L., Alakomi, H.L. and Oksman-Caldentey, K. M. (2005). Bioactive berry compounds - nove tools against human pathogens. Applied Microbiology and Biotechnology 67, 8-18.

Puupponen-Pimiä, R., Nohynek, L., Hartmann-Schmidlin, S., Kähkönen, M., Heinonen, M., Maatta-Riihinen, K. and Oksman-Caldentey, K. M. (2005). Berry phenolics selectively inhibit the growth of intestinal pathogens. Fournal of Applied Microbiology $\mathbf{9 8}$ 991-1000
Puupponen-Pimiä, R., Nohynek, L., Meier, C., Kähkönen, M., Heinonen, M., Hopia, A. and Oksman-Caldentey, K.-M. (2001) Antimicrobial properties of phenolic compounds from berries. Fournal of Applied Microbiology 90, 494-507.

Rossignol, J.F. (2010). Cryptosporidium and Giardia: treatment options and prospects for new drugs. Experimental Parasitology 124, 45-53.

Shuaibu, M. N., Wuyep, P.T.A., Yanagi, T., Hirayama, K., Ichinose, A., Tanaka, T. and Kouno, I. (2008). Trypanocidal activity of extracts and compounds from the stem bark of Anogeissus leiocarpus and Terminalia avicennoides. Parasitology Research 102, 697-703.

Soh, P. N., Witkowski, B., Olagnier, D., Nicolau, M. L., Berry, A. and Benoit-Vical, F. (2009). In vitro and in vivo properties of ellagic acid in malaria treatment. Antimicrobial Agents and Chemotherapy 53, 1100-1106.

Soffar, S. A. and Mokhtar, G. M. (1991). Evaluation of the antiparasitic effect of aqueous garlic (Allium sativum) extract in Hymenolepiasis nana and giardiasis. Fournal of the Egyptian Society of Parasitology 21, 497-502.

Upcroft, J. A., Dunn, L. A., Wright, J. M., Benakli, K., Upcroft, P. and Vanelle, P. (2006). 5-Nitroimidazole drugs effective against metronidazoleresistant Trichomonas vaginalis and Giardia duodenalis. Antimicrobial Agents and Chemotherapy 50, 344-347.

Yamasaki, T., Sato, M., Mori, T., Mohamed, A. S. A., Fujii, K. and Tsukioka, J. (2002). Toxicity of tannins towards the free-living nematode Caenorhabditis elegans and the brine shrimp Artemia salina. Fournal of Natural Toxins 11, 165-171.

Yoshida, T., Hatano, T., Ito, H. and Okuda, T. (2009). Structural diversity and antimicrobial activities of ellagitannins. In Chemistry and Biology of Ellagitannins: An Underestimated Class of Bioactive Plant Polyphenols (ed. Quideau, S.), pp. 55-93. World Scientific Publishing, London, UK. 